Results Five cytokines (GM-CSF, IL-1 $\beta$, IL-4, IL-13, IL-17E/ $25)$ returned $<20 \%$ concentrations within detection limits and were excluded from further analysis. The other cytokines returned $>85 \%$ samples in range. IL-6, IL-8 and IL-10 increased after exercise (IL-6: $F=36, \mathrm{p}<0.0001$; IL-8: $F=39$, $\mathrm{p}<0.0001$; IL-10: $F(1,21)=8.9, \mathrm{p}=0.0072)$. There was a trend towards a greater post-exercise increase in IL-10 with HME (HME: median 0.062 (range $-0.203-1.053$ ) pg/mL; no-HME: $0.047(-0.079-0.50) \mathrm{pg} / \mathrm{mL} ; F=3.0, \mathrm{p}=0.096)$. There were no significant interactions for other cytokines.

Conclusions Use of an HME during exercise in a sub-zero environment did not affect systemic pro- and anti-inflammatory cytokine responses to exercise. Local inflammatory markers in the lungs may be relevant to investigate in future studies.

\section{A COMPARISON OF THE PREVALENCE AND SEVERITY OF HEALTH PROBLEMS IN NATIONAL TEAM CROSS- COUNTRY SKIERS OVER A COMPETITIVE SEASON}

${ }^{1}$ Øyvind Karlsson, ${ }^{2}$ Magnus Danemar, 'Marko S Laaksonen, 'Kerry McGawley. 'Swedish Winter Sports Research Centre, Department of Health Sciences, Mid Sweden University, Östersund, Sweden; 'Department of Physiology and Pharmacology, Karolinska Institutet, Stockholm, Sweden

\subsection{6/bjsports-2021-IOC.357}

Background A limited number of long-term prospective studies have investigated health problems in competitive cross-country skiers. Moreover, limited information exists regarding differences in the prevalence and severity of health problems between senior and development female and male cross-country skiers. Objective To compare the prevalence and severity of health problems between senior and development female and male national team cross-country skiers.

Design Comparative, prospective, observational cohort study.

Setting Senior and development national cross-country ski teams.

Participants Eighteen (9 women, 9 men) senior and 22 (9 women, 13 men) development national team skiers.

Intervention Skiers self-reported their health problems weekly for 17 weeks throughout the 2019/2020 competitive crosscountry ski season using the Oslo Sports Trauma Research Center Questionnaire on Health Problems (OSTRC-H2).

Main Outcome Measurements We calculated weekly prevalence and severity measures for illnesses, acute and overuse injuries, substantial health problems (i.e., health problems leading to moderate or severe reductions in training volume or sports performance, or a complete inability to participate) and all health problems combined.

Results Weekly response rate to the OSTRC-H2 was 90\% (SD $7 \%)$. Average weekly prevalence of substantial $(\sim 12 \%)$ and all $(\sim 25 \%)$ health problems were similar between senior and development team skiers. However, illness prevalence was lower in senior vs. development skiers $(8 \%, 95 \%$ CI $[3 \%$, $13 \%]$ vs. $13 \%, 95 \%$ CI $[9 \%, 17 \%]$, respectively; $p=.026)$ and injuries were higher $(12 \%, 95 \%$ CI $[9 \%, 15 \%]$ vs. $5 \%$, $95 \%$ CI $[3 \%, 7 \%] ; \mathrm{p}<.001)$. There were no differences in severity measures (all $\mathrm{p}>.05$ ).

Conclusions Health problems were relatively common among the skiers, with one quarter affected by at least one problem at any given time. While the prevalence of all health problems was similar, illnesses were less prevalent in the senior compared to the development team skiers and injuries were more prevalent.

\section{THE PREVALENCE AND BURDEN OF HEALTH PROBLEMS IN MALE SENIOR ICE HOCKEY: A PROSPECTIVE STUDY IN THE NORWEGIAN PROFESSIONAL LEAGUE}

${ }^{1,2}$ Anine Nordstrøm, ${ }^{1}$ Roald Bahr, ${ }^{2}$ Ove Talsnes, ${ }^{1}$ Benjamin Clarsen. ${ }^{1}$ Department of Sports Medicine, Oslo Sports Trauma Research Center, Norwegian School of Sports Sciences, Oslo, Norway; ${ }^{2}$ Innlandet Hospital Trust, Elverum, Norway

\section{$10.1136 /$ bjsports-2021-IOC.358}

Background As previous epidemiological studies in elite ice hockey have focused on acute time-loss injuries, little is known about the burden of overuse injuries and illnesses in ice hockey.

Objective The aim of this study was to report the prevalence and burden of all health problems in male senior elite ice hockey during one competitive season.

Design Prospective cohort study.

Setting The 2017/2018 competitive season of the GET league (the premier professional league) in Norway.

Patients (or Participants) We included nine of the ten teams in the GET league (the premier professional league) in Norway. In the nine teams participating, five players declined to participate, six players did not report and one player dropped out. A total of 225 players finished the study.

Interventions (or Assessment of Risk Factors) Players reported all health problems; acute injuries, overuse injuries and illnesses, for 31 weeks using Oslo Sports Trauma Research Center Questionnaire on Health Problems.

Main Outcome Measurements Prevalence and burden of acute injuries, overuse injuries and illnesses.

Results At any given time, 40\% (95\% CI 37\% to $43 \%$ ) reported symptoms from injury or illness, and 20\% $95 \% \mathrm{CI}$ $19 \%$ to $22 \%$ ) experienced health problems with a substantial negative impact on training and performance. Acute injuries represented the greatest incidence, prevalence and burden. The most burdensome acute injuries were to the head/face, shoulder, knee and ankle. The most burdensome overuse injury locations were knee, lumbar spine and hip/groin.

Conclusions This registration did capture a greater burden from overuse injuries than traditional injury registration, but acute injuries did represent the major problem.

\section{PROMOTION FOR JAPAN SKIING SAFETY}

Yukio Urabe, Kazuki Fukui, Junpei Sasadai, Makoto Komiya, Masanori Morikawa, Noriaki Maeda. Graduate School of Biomedical and Health Sciences, Hiroshima University, Hiroshima, Japan

\subsection{6/bjsports-2021-IOC.359}

Background Niseko, which is located north of Japan, becomes a famous ski resort for the best powder snow called as 'JAPOW' or 'NISEPOW'. As the number of backcountry skiing's popularity increased, accidents related skiing also did in Niseko.

Objective The purpose was to show the effort for the safety of skiers in Niseko and how to prevent and decrease the number of ski accidents in Japan.

Design Cross-sectional study.

Setting Field of Niseko skiing resort. 